\title{
Editorial: Data-driven modelling and high-fidelity simulations
}

\section{Original article}

Article history:

Submission date: 9 April 2021

Acceptance date: 20 April 2021

Publication date: 25 May 2021

Check for updates

*Correspondence:

VM: vittorio.michelassi@bakerhughes.com

\section{Copyright:}

(c) 2021 Baker Hughes Company LLC @ (This is an open access article distributed under the Creative Commons Attribution Non Commercial No Derivatives License (CC BY-NC-ND 4.0). Unrestricted use, distribution, and reproduction of the original work are permitted for noncommercial purposes only, provided it is properly cited and its authors credited. No derivative of this work may be distributed.

\section{Keywords:}

turbines; compressors; machine learning; high-fidelity simulations

\section{Citation:}

Michelassi V., He L. (2021). Editorial: Datadriven modelling and high-fidelity simulations. Journal of the Global Power and Propulsion Society. Special Issue: DataDriven Modelling and High-Fidelity Simulations: $1-2$. https://doi.org/10.33737/jgpps/135933
Vittorio Michelassi ${ }^{1,{ }^{*}}, \mathrm{Li} \mathrm{He}$

${ }^{1}$ Baker Hughes, Via Matteucci 2, 50127 Firenze, Italy

${ }^{2}$ Oxford University, Parks Road, Oxford, OX1 3PJ, UK

Design of gas turbine turbomachinery components requires the contribution from multiple disciplines: materials, mechanical, thermal, aerodynamics, rotor-dynamics, operability, emissions and many others. The development of corresponding design and verification methods and tools has enabled the evolution of performance including efficiency, reliability, cost, operability and manufacturability. Still, the scope of gas-turbine technology development is strongly influenced (as well as limited) by the quality and capability of the conventional design methods and tools.

The special issue of the GPPS Journal was initiated against the backdrop of both the rapid advances in Artificial Intelligence (AI) and Machine Learning (ML) in many fields globally in general, and the more specific needs and challenges in gas turbine technology development in particular. The papers selected should be indicative of the recent advances in design and verification methods that take advantage of the continuous growth of computational resources, the availability of large data sets, and the evolution of methods and algorithms. The new methods and new understanding gained should offer new opportunities to unlock further improvements and new technology development outside conventional design spaces (e.g. new fuels, interface with renewables or carbon avoidance, reduction, capture and sequestration etc). These should also possibly allow to address the two key challenges rotating machinery will face in the future decades. First, the rapidly changing scenario of power generation and aircraft propulsion requires shorter time cycle for design-to-market without compromising performance, availability, and cost. Second, the foreseeable future will see a further step-change in technology and opportunities that call for significant extension of the design space and decisive nonconventional steps away from current design comfort-zones.

Regardless of possible forms and shapes that the future energy conversion systems will take (based on rotating machinery or not), it should be reasonable to assume that they will still go in parallel with the evolution of design and verification methods. The validity, applicability range and accuracy level of design tools must be improved, as design uncertainties translate into design safety margins and ultimately sub-optimal performance. The "digital-twin" exemplifies this trend, in which every physical asset is paired with its digital counterpart. Nevertheless, it is the absolute accuracy of the digital models that will need to be further improved to be able to offer new solutions that are not based on previous experience. Ultimately, designers will develop new products with these advanced tools of multiple fidelities from the very early design up to the final detailed design phase. 
The contributors to the special issue are invited to give both academia and industry perspectives on new development trend in design methods. High-fidelity simulations can offer both a close-up lens to shed light on very fundamental aerothermal physics, and a powerful and reliable tool to investigate complex component-to-component interactions. The very large simulation data sets require new postprocessing techniques, readily applicable also to experimental data sets coming from dedicated tests or from field operations, to capture trends and suggest physical interpretations that may guide design improvements. Units are constantly monitored with numerous probes, and the large data sets gained can inform on deviation from optimal utilization and possible anomalies. The availability of large data sets, regardless whether they come from simulations or from measurements, requires the assessment of their quality and uncertainty. The tremendous opportunities offered by the range of new methods are already suggesting innovative solutions that need to be paralleled by both new materials and manufacturing techniques.

The collection of the contributions to this special issue covers some of these key topics. We hope that the readers will find these articles informative and interesting, to stimulate further development and applications in power generation and energy conversion that will alleviate the problems associated with the energy demand and the associated environmental impact.

\section{Competing interests}

Vittorio Michelassi declares that he has no conflict of interest. Li He declares that he has no conflict of interest. 